\title{
A Generic Camera Calibration Method for Fish-Eye Lenses
}

\author{
Juho Kannala and Sami Brandt \\ Helsinki University of Technology \\ Laboratory of Computational Engineering \\ P.O. Box 9203, 02015 HUT, Finland \\ jkannala@1ce.hut.fi, Sami.Brandt@hut.fi
}

\begin{abstract}
Fish-eye lenses are convenient in such computer vision applications where a very wide angle of view is needed. However, their use for measurement purposes is limited by the lack of an accurate, generic, and easy-to-use calibration procedure. We hence propose a generic camera model for cameras equipped with fish-eye lenses and a method for calibration of such cameras. The calibration is possible by using only one view of a planar calibration object but more views should be used for better results. The proposed calibration method was evaluated with real images and the obtained results are promising. The calibration software will become commonly available at the author's Web page.
\end{abstract}

\section{Introduction}

Cameras with a narrow-angle lens can be modelled with the pinhole camera model which is just a perspective projection followed by an affine transformation in the image plane [5]. The fish-eye lens instead is designed to cover the whole hemispherical fi eld in front of the camera, hence, the angle of view is very large, about $180^{\circ}$. Because it is impossible to project the hemispherical field of view on a finite image plane by a perspective projection, fish-eye lenses are designed to obey some other projection model. Therefore the inherent distortion of a fish-eye lens should not be considered only as a deviation from the pinhole model [7].

There have been some efforts to model the radially symmetric distortion of fish-eye lenses with different models $[2,4,3]$. The idea of these approaches is to transform the original fi sh-eye image to follow the pinhole model. In [4] and [3], the parameters of the distortion model are estimated by forcing that straight lines are straight after the transformation but the problem is that the methods do not give the full calibration. They can be used to "correct" the images to follow the pinhole model but their applicability is limited when one needs to know the direction of a back-projected ray corresponding to an image point. The calibration procedures by Shah and Aggarwal [8] and Bakstein and $\mathrm{Pa}-$ jdla [1] instead aim at calibrating fish-eye lenses generally. However, their methods are slightly cumbersome in practise because a laser beam or a cylindrical calibration object is required.

In this paper, we propose a new general calibration method that requires that the camera observes a planar calibration pattern. More precisely, in Section 2, we describe the camera model that we have used for fi sh-eye lens cameras. In Section 3, we propose a procedure for estimating the parameters of the model, and the results are presented and discussed in Sections 4 and 5.

\section{Camera model}

\subsection{Generic model for a fish-eye lens camera}

The perspective projection of a pinhole camera can be described by the following formula

$$
r=f \tan \theta \quad \text { (perspective projection), }
$$

where $\theta$ is the angle between the optical axis and the incoming ray, $r$ is the distance between the image point and the principal point and $f$ is the focal length. Fish-eye lenses instead are usually designed to obey one of the following projections:

$$
\begin{aligned}
& r=2 f \tan (\theta / 2) \quad \text { (stereographic projection), } \\
& r=f \theta \quad \text { (equidistance projection), } \\
& r=2 f \sin (\theta / 2) \quad \text { (equisolid angle projection), } \\
& r=f \sin \theta \quad \text { (orthogonal projection). }
\end{aligned}
$$

Perhaps the most common model is the equidistance projection. The behaviour of the different projections is illustrated in Fig. 1(a) and the difference between a pinhole camera and a fi sh-eye camera is shown in Fig. 1(b).

The real lenses do not exactly follow the designed projection model. From the viewpoint of automatic calibration, it would also be useful if we had only one model for different types of fish-eye lenses. Therefore we consider projections in the general form

$$
r(\theta)=k_{1} \theta+k_{2} \theta^{3}+k_{3} \theta^{5}+k_{4} \theta^{7}+\ldots,
$$




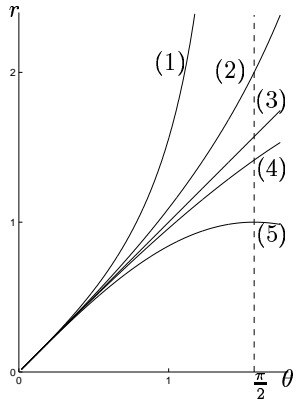

(a)

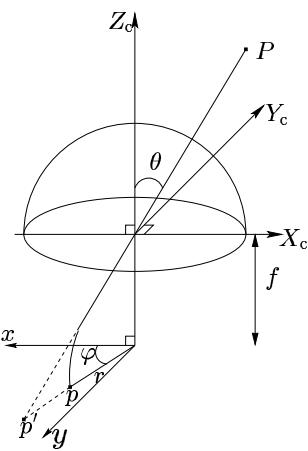

(b)
Figure 1: (a) Curves of projections (1)-(5) with $f=1$. (b) Fisheye camera model. The image of the point $P$ is $p$ whereas it would be $p^{\prime}$ by a pinhole camera.

where, without any loss of generality, even powers have been dropped. This is due that we may extend $r$ onto the negative side as an odd function while the odd powers span the set of continuous odd functions.

For computations we need to fix the number of terms in (6). An important property for a projection model is that it can be analytically inverted. Therefore we choose

$$
r(\theta)=k_{1} \theta+k_{2} \theta^{3}
$$

as the basic model. When modelling real lenses, the values of parameters $k_{1}$ and $k_{2}$ will be such that $r(\theta)$ is monotonically increasing on the interval $[0, \pi / 2]$. Therefore we may solve $\theta$ from (7) if $r$ is given: from the three possible roots to a cubic equation we choose a real root that is between 0 and $\pi / 2$. Although the model (7) contains only two parameters, it can approximate all the projections (2)-(5) with a moderate level of accuracy. The difference would be hardly distinguishable if the approximation nearest in $L^{2}$-norm would be plotted to Fig. 1(a) for each projection.

We additionally need to transform the camera coordinates $\left(X_{\mathrm{c}}, Y_{\mathrm{c}}, Z_{\mathrm{c}}\right) \hat{=}(\rho, \varphi, \theta)$ into the image pixel coordinates. As an intermediate step we compute the normalised image coordinates (see Fig. 1(b))

$$
\left(\begin{array}{l}
x \\
y
\end{array}\right)=r(\theta)\left(\begin{array}{c}
\cos \varphi \\
\sin \varphi
\end{array}\right)
$$

where $r(\theta)$ is obtained from (7). By assuming that the pixel coordinate system is orthogonal we get the pixel coordinates $(u, v)$ from

$$
\left(\begin{array}{l}
u \\
v
\end{array}\right)=\left[\begin{array}{cc}
m_{u} & 0 \\
0 & m_{v}
\end{array}\right]\left(\begin{array}{l}
x \\
y
\end{array}\right)+\left(\begin{array}{l}
u_{0} \\
v_{0}
\end{array}\right)
$$

where $\left(u_{0}, v_{0}\right)$ is the principal point and $m_{u}$ and $m_{v}$ give the number of pixels per unit distance in horizontal and vertical directions, respectively.

\subsection{Extended model with distortion}

The lens elements of a real fish-eye lens may deviate from precise radial symmetry and they may be inaccurately po- sitioned causing that the projection is not exactly radially symmetric. We hence propose adding two distortion terms: one acting in the radial direction

$$
\begin{aligned}
\Delta_{r}(\theta, \varphi)= & \left(l_{1} \theta+l_{2} \theta^{3}+l_{3} \theta^{5}\right)\left(i_{1} \cos \varphi+\right. \\
& \left.i_{2} \sin \varphi+i_{3} \cos 2 \varphi+i_{4} \sin 2 \varphi\right),
\end{aligned}
$$

and the other in the tangential direction

$$
\begin{aligned}
\Delta_{t}(\theta, \varphi)= & \left(m_{1} \theta+m_{2} \theta^{3}+m_{3} \theta^{5}\right)\left(j_{1} \cos \varphi+\right. \\
& \left.j_{2} \sin \varphi+j_{3} \cos 2 \varphi+j_{4} \sin 2 \varphi\right) .
\end{aligned}
$$

The distortion functions are thus separable in the variables $\theta$ and $\varphi$. Because the Fourier series of any $2 \pi$-periodic continuous function converges in $L^{2}$-norm and any continuous odd function can be represented by a series of odd polynomials we can, in principle, model any kind of continuous distortion by simply adding more terms to (10) and (11).

With the distortion parameters we get the following formula for the normalised image coordinates

$$
\left(\begin{array}{l}
x \\
y
\end{array}\right)=\left(r(\theta)+\Delta_{r}(\theta, \varphi)\right) \mathbf{u}_{r}(\varphi)+\Delta_{t}(\theta, \varphi) \mathbf{u}_{\varphi}(\varphi)
$$

where $\mathbf{u}_{r}(\varphi)$ and $\mathbf{u}_{\varphi}(\varphi)$ are the unit vectors in the radial and tangential directions. Since the analytical invertibility of the model (8) is anyway lost in (12), we may also take more parameters to the radially symmetric part $r(\theta)$. Using the model (6) with terms up to the ninth order increases the total number of parameters to 23 .

\section{Calibration method}

The basic model introduced in Section 2.1 contains the six internal camera parameters $k_{1}, k_{2}, m_{u}, m_{v}, u_{0}$ and $v_{0}$. We determine the parameter values by viewing a calibration plane which contains control points in known positions. In Section 3.1, we propose a four step procedure for the calibration when $M$ control points are observed in $N$ views. In Section 3.2, we suggest a modification to the method when the control points are circular.

\subsection{Four step procedure}

For each view, there is a rotation matrix $\mathbf{R}^{j}$ and a translation vector $\mathbf{t}^{j}$ describing the position of the camera with respect to the calibration plane such that

$$
\mathbf{X}_{\mathrm{c}}^{j}=\mathbf{R}^{j} \mathbf{X}+\mathbf{t}^{j}, \quad j=1, \ldots, N .
$$

We choose the calibration plane to lie in the $X Y$-plane and denote the coordinates of control point $i$ with $\mathbf{X}_{i}=$ $\left(X_{i}, Y_{i}, 0\right)^{\top}$. The corresponding homogeneous coordinates in the calibration plane are denoted by $\mathbf{x}_{i}^{\mathbf{p}}=$ $\left(X_{i}, Y_{i}, 1\right)^{\top}$ and the observed coordinates in view $j$ by $\mathbf{m}_{i}^{j}=\left(u_{i}^{j}, v_{i}^{j}, 1\right)^{\top}$. For the internal parameters we use a short-hand notation $\mathbf{p}=\left(k_{1}, k_{2}, m_{u}, m_{v}, u_{0}, v_{0}\right)$. 


\section{Step 1: Initialisation of internal parameters}

The initial guesses for $k_{1}$ and $k_{2}$ are obtained by fitting (7) to the desired projection (2)-(5) with the manufacturer's values for the nominal focal length $f_{0}$ and the angle of view $\theta_{\max }$. Then we also obtain the radius of the image on the sensor plane by $r_{\max }=k_{1} \theta_{\max }+k_{2} \theta_{\max }^{3}$.

With a circular image fi sh-eye lens, the actual image fills only a circular area inside the image frames. In pixel coordinates, this circle is an ellipse

$$
\left(\frac{u-u_{0}}{a}\right)^{2}+\left(\frac{v-v_{0}}{b}\right)^{2}=1,
$$

whose parameters can be estimated. Consequently, we obtain initial guesses for the remaining unknowns $m_{u}, m_{v}$, $u_{0}$, and $v_{0}$ in $\mathbf{p}$, where $m_{u}=a / r_{\max }$ and $m_{v}=b / r_{\max }$. With a full-frame fish-eye lens, the best thing is probably to place the principal point to the image centre and use the reported values of the pixel dimensions to obtain initial values for $m_{u}$ and $m_{v}$.

\section{Step 2: Refinement of the internal parameters}

With the internal parameters $\mathbf{p}$, we transform the observed points $\mathbf{m}_{i}^{j}$ to points $\mathbf{x}_{i}^{\prime j}$ that approximately follow the perspective projection for each $j$ (in Fig. 1(b), this corresponds to transforming the point $p$ to $p^{\prime}$ ). Under perspective imaging, the mapping between the calibration plane and the image plane is a planar homography for which holds $\mathbf{x}_{i}^{\prime j}=\mathbf{H}^{j} \mathbf{x}_{i}^{\mathbf{p}}$. The aim of this step is to iteratively search such parameter values that the mapping between $\mathbf{x}_{i}^{\mathrm{p}}$ :s and $\mathbf{x}_{i}^{\prime j}$ :s is as close to a homography as possible.

In practise, we suggest the following scheme for computing the error vector $\epsilon=\mathcal{F}(\mathbf{p})$, where $\mathcal{F}: \mathbb{R}^{6} \rightarrow \mathbb{R}^{N M}$.

(i) Back-project the control points by fi rst computing the normalised image coordinates

$$
\left(\begin{array}{c}
x_{i}^{j} \\
y_{i}^{j}
\end{array}\right)=\left[\begin{array}{cc}
1 / m_{u} & 0 \\
0 & 1 / m_{v}
\end{array}\right]\left(\begin{array}{l}
u_{i}^{j}-u_{0} \\
v_{i}^{j}-v_{0}
\end{array}\right),
$$

transforming them to the polar coordinates $\left(r_{i}^{j}, \varphi_{i}^{j}\right) \hat{=}\left(x_{i}^{j}, y_{i}^{j}\right)$, and fi nally computing $\theta_{i}^{j}$ from (7).

(ii) Re-project the rays $\left(\theta_{i}^{j}, \varphi_{i}^{j}\right)$ using (1) with $f=1$ to obtain the points $\mathbf{x}_{i}^{\prime}$.

(iii) Compute the homography estimates $\hat{\mathbf{H}}^{j}$ from the correspondences $\mathbf{x}_{i}^{\prime j} \leftrightarrow \mathbf{x}_{i}^{\mathrm{p}}$ by the linear algorithm with data normalisation [5]. Defi ne $\hat{\mathbf{x}}_{i}^{j}$ as the exact image of $\mathbf{x}_{i}^{\mathbf{p}}$ under $\hat{\mathbf{H}}^{j}$ such that $\hat{\mathbf{x}}_{i}^{j}=\hat{\mathbf{H}}^{j} \mathbf{x}_{i}^{\mathrm{p}}$.

(iv) Compute the distances $\epsilon_{i}^{j}=d\left(\mathbf{x}_{i}^{\prime j}, \hat{\mathbf{x}}_{i}^{j}\right)$, combine them to vectors $\boldsymbol{\epsilon}^{j}=\left(\epsilon_{1}^{j}, \ldots, \epsilon_{M}^{j}\right)$ and further to a single vector $\boldsymbol{\epsilon}=\left(\boldsymbol{\epsilon}^{1}, \ldots, \boldsymbol{\epsilon}^{N}\right)$.

We then use the Levenberg-Marquardt algorithm to minimise $\|\boldsymbol{\epsilon}\|$ with respect to $\mathbf{p}$.

\section{Step 3: Initialisation of external parameters}

First we refi ne the homographies $\mathbf{H}^{j}$ by minimising the errors $\left\|\boldsymbol{\epsilon}^{j}\right\|$ while keeping $\mathbf{p}$ fi xed. Then, perspective imaging of the calibration plane, with $f=1$, gives

$$
s\left(\begin{array}{c}
x_{i}^{\prime j} \\
y_{i}^{\prime j} \\
1
\end{array}\right)=\left[\begin{array}{ll}
\mathbf{R}^{j} & \mathbf{t}^{j}
\end{array}\right]\left(\begin{array}{c}
X_{i} \\
Y_{i} \\
0 \\
1
\end{array}\right)=\left[\begin{array}{lll}
\mathbf{r}_{1}^{j} & \mathbf{r}_{2}^{j} & \mathbf{t}^{j}
\end{array}\right]\left(\begin{array}{c}
X_{i} \\
Y_{i} \\
1
\end{array}\right)
$$

which implies $\mathbf{H}^{j}=\left[\mathbf{r}_{1}^{j} \mathbf{r}_{2}^{j} \mathbf{t}^{j}\right]$, up to scale. Furthermore

$$
\begin{aligned}
\mathbf{r}_{1}^{j}=\lambda^{j} \mathbf{h}_{1}^{j}, \quad \mathbf{r}_{2}^{j}=\lambda^{j} \mathbf{h}_{2}^{j}, \quad \mathbf{r}_{3}^{j}=\mathbf{r}_{1}^{j} \times \mathbf{r}_{2}^{j}, \\
\mathbf{t}^{j}=\lambda^{j} \mathbf{h}_{3}^{j},
\end{aligned}
$$

where $\lambda^{j}=\operatorname{sign}\left(h_{3,3}^{j}\right) /\left\|\mathbf{h}_{1}^{j}\right\|$. Because of estimation errors, the obtained rotation matrices are not orthogonal. Thus we use the singular value decomposition to compute the closest orthogonal matrices in the sense of Frobenius norm [9] and use them as initial guess for each $\mathbf{R}^{j}$.

\section{Step 4: Minimisation of projection error}

As we have the estimates for the internal and external camera parameters, we use (13), (8) or (12), and (9) to compute the imaging function $\mathcal{P}^{j}$ for each camera, where a control point is projected to $\hat{\mathbf{m}}_{i}^{j}=\mathcal{P}^{j}\left(\mathbf{X}_{i}\right)$. The camera parameters are refined by minimising the sum of squared distances between the measured and modelled control point projections

$$
\sum_{j=1}^{N} \sum_{i=1}^{M} d\left(\mathbf{m}_{i}^{j}, \hat{\mathbf{m}}_{i}^{j}\right)^{2}
$$

using the Levenberg-Marquardt algorithm. If (12) is used instead, the additional parameters may be initialised to zero.

\subsection{Modification for circular control points}

In order to achieve an accurate calibration, we used a calibration plane with white circles on black background since the centroids of the projected circles can be detected with a sub-pixel level of accuracy [6]. In this setting, however, the problem is that the centroid of the projected circle is not the image of the centre of the original circle. Therefore, since $\mathbf{m}_{i}^{j}$ in (14) is the measured centroid, we should not project the centres as points $\hat{\mathbf{m}}_{i}^{j}$.

To avoid the problem above, we propose solving the centroids of the projected circles numerically. We parameterise the interior of the circle at $\left(X_{0}, Y_{0}\right)$ with radius $R$ by $\mathbf{X}(\varrho, \alpha)=\left(X_{0}+\varrho \sin (\alpha), Y_{0}+\varrho \cos (\alpha), 0\right)^{\top}$. Given the camera parameters, we get the centroid $\hat{\mathbf{m}}$ for the circle by numerically evaluating

$$
\hat{\mathbf{m}}=\frac{\int_{0}^{R} \int_{0}^{2 \pi} \hat{\mathbf{m}}(\varrho, \alpha)|\operatorname{det} \mathbf{J}(\varrho, \alpha)| d \alpha d \varrho}{\int_{0}^{R} \int_{0}^{2 \pi}|\operatorname{det} \mathbf{J}(\varrho, \alpha)| d \alpha d \varrho},
$$

where $\hat{\mathbf{m}}(\varrho, \alpha)=\mathcal{P}(\mathbf{X}(\varrho, \alpha))$ and $\mathbf{J}(\varrho, \alpha)$ is the Jacobian of the composite function $\mathcal{P} \circ \mathbf{X}$. The analytical solving of the Jacobian is rather a tedious task but it can be computed by mathematical software such as Maple. 


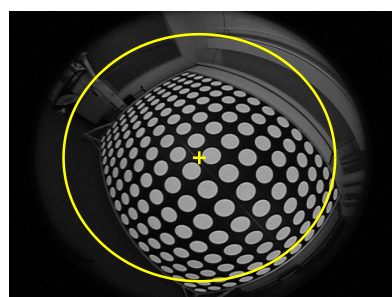

(a) (b)

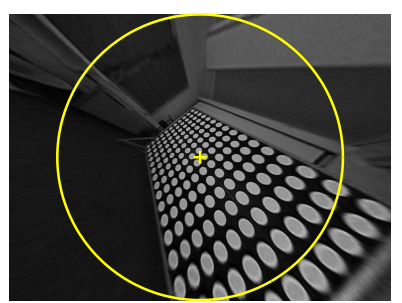

Figure 2: Fish-eye lens calibration using only one view. (a) Original image where the ellipse depicts the fi eld of view of $150^{\circ}$. (b) The image corrected to follow pinhole model. Straight lines are straight as they should be.

\section{Experiments}

In the experiments, we used an equidistance fish-eye lens with the nominal focal length of $1.178 \mathrm{~mm}$ attached to a Watec 221S CCD colour camera. The calibration object was a $2 \times 3 \mathrm{~m}^{2}$ plane containing white circles with the radius of 60 $\mathrm{mm}$ on the black background. The calibration images were digitised from an analog video signal to 8-bit monochrome images, whose size was 640 by 480 pixels.

When the basic model (8) is used the calibration can be performed even from a single image of the planar object as Fig. 2 illustrates. In that example we used 60 control points for the calibration. However, for the most accurate results, the whole fi eld of view should be covered with a large number of measurements. Therefore we experimented our method with 12 views and 740 points in total with varying number of internal parameters in the final calibration step. The results are in Table 1. It can be seen that the centroid correction has a very important role. The extended model (12) gives the smallest deviations $\sigma_{u}$ and $\sigma_{v}$ in the $x$ and $y$ directions, respectively, but the basic model with the three additional parameters gives almost as good results. Nevertheless, there should be no risk of over- fitting because the number of measurements is large. The estimated asymmetric distortion and the residuals are displayed in Fig. 3.

To demonstrate the achieved level of accuracy in another way, we approximate the size of the solid angle that projects to an ellipse with principal axes $2 \sigma_{u}$ and $2 \sigma_{v}$. For the equidistance projection the solid angle corresponding to the small area $d S$ in the image plane is

$$
d \Omega=\frac{1}{f^{2}} \frac{\sin \theta}{\theta} d S .
$$

Near the principal point $(\theta=0)$ with $f \approx k_{1}=1.12 \mathrm{~mm}$ we

Table 1: Standard deviation of the residuals $(\mathbf{m}-\hat{\mathbf{m}})$ where $\mathbf{p}_{6}$ is the basic model, $\mathbf{p}_{9}$ is the basic model with three additional parameters $\left(k_{3}, k_{4}, k_{5}\right)$, and $\mathbf{p}_{23}$ is the extended model. The star (*) indicates that the centroid correction of Sec. 3.2 is not used.

\begin{tabular}{|l|l|l|l|l|}
\hline & $\mathbf{p}_{6}^{*}$ & $\mathbf{p}_{6}$ & $\mathbf{p}_{9}$ & $\mathbf{p}_{23}$ \\
\hline$\sigma_{u}[\mathrm{pix}]$ & 0.26 & 0.11 & 0.074 & 0.069 \\
\hline$\sigma_{v}[\mathrm{pix}]$ & 0.24 & 0.10 & 0.060 & 0.058 \\
\hline
\end{tabular}

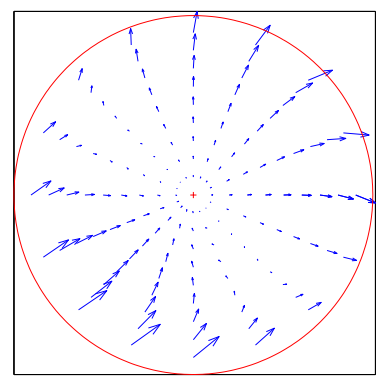

(a)

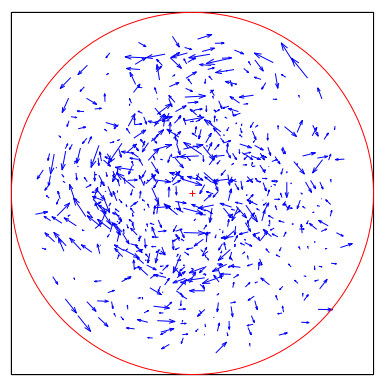

(b)
Figure 3: (a) The estimated asymmetric distortion $\left(\Delta_{r} \mathbf{u}_{r}+\Delta_{t} \mathbf{u}_{\varphi}\right)$ using the extended model $\mathbf{p}_{23}$. (b) The remaining residual for each control point that shows no obvious systematic error. Both plots are in normalised image coordinates and the vectors are scaled up by a factor of 150 to aid inspection.

have $d \Omega=4.7 \cdot 10^{-7}$. At the distance of $500 \mathrm{~mm}$ from the camera centre this corresponds approximately to the area of a circle with the radius of $0.2 \mathrm{~mm}$, which is in good agreement with the assumed accuracy of the calibration device.

\section{Conclusions}

We have proposed a novel camera calibration method for fish-eye lens cameras that is based on viewing a planar calibration pattern. The method is generic and easy-to-use, and provides for a relatively high level of accuracy by using circular control points. The achieved level of accuracy is comparable to the results of Heikkilä and Silvén for narrowangle lenses [6] that is promising considering the aim of using fish-eye lenses in measurement purposes.

\section{References}

[1] H. Bakstein and T. Pajdla. Panoramic mosaicing with a $180^{\circ}$ fi eld of view lens. In Proc. IEEE Workshop on Omnidirectional Vision, pages 60-67, 2002.

[2] A. Basu and S. Licardie. Alternative models for fi sh-eye lenses. Pattern Recognit. Lett., 16:433-441, 1995.

[3] C. Bräuer-Burchardt and K. Voss. A new algorithm to correct fish-eye- and strong wide-angle-lens-distortion from single images. In Proc. ICIP, pages 225-228, 2001.

[4] F. Devernay and O. Faugeras. Straight lines have to be straight. Mach. Vis. and Appl., 13(1):14-24, 2001.

[5] R. Hartley and A. Zisserman. Multiple view geometry in computer vision. Cambridge, 2000.

[6] J. Heikkilä and O. Silvén. Calibration procedure for short focal length off-the-shelf CCD cameras. In Proc. CVPR, pages 166-170, 1996.

[7] K. Miyamoto. Fish eye lens. J. Opt. Soc. Am., 54(8):10601061, 1964.

[8] S. Shah and J. Aggarwal. Intrinsic parameter calibration procedure for a (high-distortion) fish-eye lens camera with distortion model and accuracy estimation. Pattern Recognit., 29(11):1775-1788, 1996.

[9] Z. Zhang. A flexible new technique for camera calibration. Technical Report MSR-TR-98-71, Microsoft Research, December 1998. 